\title{
Free surface shape for an ultrasonic nebuliser
}

\author{
D. R. Jenkins*
}

(Received 1 June 2001; revised 30 September 2002)

\begin{abstract}
Nebulisers are used for the delivery of fine droplets of drugs to the lungs or smaller bronchii. An ultrasonic nebuliser uses the oscillations caused by a piezoelectric crystal to produce a "fountain" in a liquid, which leads to the formation of a mist of fine particles. This work describes a model of an ultrasonic nebuliser, with the aim of demonstrating the mechanism of the fountain formation. It is shown that the oscillating ultrasound causes a mean flow in the liquid due to acoustic streaming, which in turn creates a deformation of the free surface. Cavitation within the liquid, which is an energy intensive process, leads to the dissipation necessary for acoustic streaming to occur. Finally, a mechanism for explaining the location of the mist on the free surface is proposed, based upon the results.
\end{abstract}

${ }^{*}$ CSIRO Mathematical and Information Sciences, Locked Bag 17, North Ryde NSW 1670, AUstralia. mailto:David.R. Jenkins@csiro.au

${ }^{0}$ See http://anziamj.austms.org.au/V44/CTAC2001/Jenk for this article, (c) Austral. Mathematical Soc. 2003. Published 1 April 2003. ISSN 1446-8735 


\section{Contents}

1 Introduction

C465

2 Model equations

C467

3 Numerical solution and results

C470

4 Conclusion

C476

References

C477

\section{Introduction}

Nebulisers are used for the delivery of fine droplets of drugs to the lungs or smaller bronchii. The droplet size needs to be in the range $2-5 \mu \mathrm{m}$ for effective delivery. Traditionally, nebulisers atomise a drug by passing an air jet past a small nozzle containing a liquid. The ultrasonic nebuliser is an alternative that uses a piezoelectric crystal to produce a "fountain" in the liquid, which leads to the formation of a mist of fine particles. Ultrasonic nebulisers are less frequently used for pulmonary drug delivery than jet nebulisers, partly due to the lack of understanding of the mechanism of aerosol generation [1].

A particular type of ultrasonic nebuliser was considered at the 1998 Mathematics in Industry Study Group [2] (MISG) with the aim of understanding the mechanism of the fountain formation. The fountain is formed by locating a curved (parabolic) piezoelectric crystal with its focus at a point just above the free surface of the liquid. The energy in the ultrasonic waves produced within the liquid in some way causes the formation of a "splashing" fountain. A 
particular feature of the fountain observed during the MISG deliberations was that the amount of splash and intensity of the fountain was reduced as the liquid level was increased, so that its surface was above the focal point of the transducer. If the liquid level was sufficiently high, then the splashing and mist formation ceases and the fountain became an almost steady bump on the free surface. In this paper, the emphasis is on understanding the fountain formation process by examining the situation where the mean liquid surface lies above the focus of the transducer, as the liquid free surface is stable, and more likely amenable to analysis. The analysis breaks down as the liquid level is lowered, but it indicates how the steady bump becomes unstable and leads to a fountain formation. Another feature of the analysis is that it helps to explain the location of the mist formation on the liquid free surface.

The fountain formation/free surface bump will be shown to occur due to acoustic streaming, which is described most clearly by Lighthill [3]. Acoustic streaming is the development of a mean steady flow in a fluid by an acoustic (oscillatory) signal within the fluid. A crucial aspect of the effect is that an energy dissipation mechanism is required for it to occur. In this case, it is likely that the principal dissipation effect is caused by cavitation, which is known to occur in ultrasonic nebulisers [1]. The formation and collapse of bubbles that occurs in cavitation is an energy intensive process, and the focussing of the ultrasound signal leads to a significant amount of cavitation here. Moreover, cavitation has been shown experimentally to be a key mechanism in the development of acoustic streaming in liquids [4]. 


\section{Model equations}

We consider the flow of a viscous, compressible fluid, with govering equations of continuity,

$$
\frac{\partial \rho}{\partial t}+\nabla \cdot(\rho \mathbf{u})=0
$$

momentum,

$$
\rho\left[\frac{\partial \mathbf{u}}{\partial t}+\mathbf{u} \cdot \nabla \mathbf{u}\right]=-\nabla p-\rho g \hat{\mathbf{z}}+\mu \nabla^{2} \mathbf{u},
$$

and (approximate) equation of state

$$
\rho=\rho_{0}+\frac{1}{c^{2}}\left(p-p_{0}\right),
$$

where $c$ is the speed of sound in the liquid. For water at room temperature, $c \approx 1500 \mathrm{~m} / \mathrm{s}$.

As acoustic streaming is due to the nonlinear interaction of oscillating fields, we write the velocity and pressure fields as a steady component and components oscillating with the forcing frequency, $\omega=2 \pi f$, and assume that all other frequencies (harmonics) have only a small effect on the steady component:

$$
\begin{gathered}
\mathbf{u}=\mathbf{u}_{c} \cos \omega t+\mathbf{u}_{s} \sin \omega t+\mathbf{u}_{2} ; \\
p-p_{0}=\phi=\phi_{c}(r, z) \cos \omega t+\phi_{s}(r, z) \sin \omega t+\phi_{2}(r, z) .
\end{gathered}
$$

Substitute these into the equations of motion, eliminate the density and then linearise the resulting equations, matching the $\sin \omega t$ and $\cos \omega t$ terms, and get the system

$$
\frac{\omega}{c^{2}} \phi_{s}+\rho_{0} \nabla \cdot \mathbf{u}_{c}=0,
$$




$$
\begin{gathered}
-\frac{\omega}{c^{2}} \phi_{c}+\rho_{0} \nabla \cdot \mathbf{u}_{s}=0, \\
-\omega \rho_{0} \mathbf{u}_{c}=-\nabla \phi_{s}-\frac{g \phi_{s}}{c^{2}} \hat{\mathbf{z}}+\mu \nabla^{2} \mathbf{u}_{\mathbf{s}}, \\
\omega \rho_{0} \mathbf{u}_{s}=-\nabla \phi_{c}-\frac{g \phi_{s}}{c^{2}} \hat{\mathbf{z}}+\mu \nabla^{2} \mathbf{u}_{\mathbf{c}} .
\end{gathered}
$$

We then eliminate the pressure fields to obtain the following pair of coupled "Helmholtz like" equations:

$$
\begin{aligned}
& \nabla\left(\nabla \cdot \mathbf{u}_{c}\right)+\mathbf{u}_{c}=-\operatorname{Fr}_{c} \nabla \cdot \mathbf{u}_{c} \hat{\mathbf{z}}-\frac{1}{\operatorname{Re}_{c}} \nabla^{2} \mathbf{u}_{s} \\
& \nabla\left(\nabla \cdot \mathbf{u}_{s}\right)+\mathbf{u}_{s}=-\operatorname{Fr}_{c} \nabla \cdot \mathbf{u}_{s} \hat{\mathbf{z}}+\frac{1}{\operatorname{Re}_{c}} \nabla^{2} \mathbf{u}_{c} .
\end{aligned}
$$

These equations have been written in dimensionless form by scaling lengths with $L=\lambda / 2 \pi=c / \omega$, where $\lambda$ is the ultrasound wavelength, and velocities with $U=A \omega$, where $A$ is the transducer oscillation amplitude. The resulting dimensionless parameters are a Froude number

$$
\operatorname{Fr}_{c}=\frac{g}{c \omega}=\frac{g L}{c^{2}},
$$

and a Reynolds number based on the speed of sound

$$
\operatorname{Re}_{c}=\frac{c^{2}}{\omega \nu}=\frac{c L}{\nu} .
$$

The tranducer is assumed to oscillate with a normal displacement of the form $A \cos \omega t$ which leads to a velocity boundary condition of

$$
\mathbf{u} \cdot \mathbf{n}=A \omega \sin \omega t,
$$

so

$$
\mathbf{u}_{s} \cdot \mathbf{n}=1 \quad \text { and } \quad \mathbf{u}_{s} \times \mathbf{n}=\mathbf{u}_{c}=0
$$


in dimensionless units. On all other solid boundaries we set

$$
\mathbf{u}_{s}=\mathbf{u}_{c}=0,
$$

and on the free boundary we have a zero stress condition.

For a typical ultrasound frequency of $f=2 \mathrm{MHz}$, we get $\mathrm{Fr}_{c} \approx$ $10^{-9} \ll 1$. For water, $\nu \approx 10^{-6} \mathrm{~m}^{2} / \mathrm{s}$, which makes $\operatorname{Re}_{c} \approx 10^{5}$. Consequently, the right hand sides of (8) and (9) should be negligible. However, we retain the viscous terms, as a representation of the sum total of dissipation of energy. Dissipation can occur from turbulence and cavitation, as well as viscous dissipation. Turbulence may or may not be relevant, but cavitation is certain to be relevant. Cavitation causes a lot of dissipation but it is too complicated to model in detail. Instead, we increase the viscosity as a model of the combined dissipative effects of cavitation and viscosity.

The time-independent terms in the momentum and continuity equations, scaled as before, and with $\phi_{s, c}$ scaled with $\rho_{0} U c$ and $\phi_{2}$ scaled with $\rho_{0} U^{2}$, give

$$
\begin{aligned}
\nabla \cdot \mathbf{u}_{2}= & 0, \\
\mathbf{u}_{2} \cdot \nabla \mathbf{u}_{2}= & -\nabla \phi_{2}-\operatorname{Fr} \hat{\mathbf{z}}+\frac{1}{\operatorname{Re}} \nabla^{2} \mathbf{u}_{2}-\frac{1}{2} \mathbf{u}_{c} \cdot \nabla \mathbf{u}_{c} \\
& -\frac{1}{2} \mathbf{u}_{s} \cdot \nabla \mathbf{u}_{s}+\frac{1}{2} \phi_{s} \mathbf{u}_{s}-\frac{1}{2} \phi_{c} \mathbf{u}_{c},
\end{aligned}
$$

where

$$
\mathrm{Fr}=\frac{g c}{U^{2} \omega}=\frac{g L}{U^{2}}
$$

is a Froude number based on $U$, and

$$
\operatorname{Re}=\frac{U c}{\omega \nu}=\frac{U L}{\nu}
$$

is a Reynolds number based on $U$. Both Fr and Re are closer to unity than their counterparts based on $c$ and cannot be neglected 
here. Terms of $\mathcal{O}\left(\mathrm{Fr}_{c}\right)$ and $\mathcal{O}(U / c)$ have been neglected as they are exceedingly small. These are the equations for the steady flow of an incompressible fluid due to the nonlinear interactions of the oscillations caused by the ultrasound.

The boundary conditions here are no slip on the solid surfaces and stress free on the free surface.

\section{$3 \quad$ Numerical solution and results}

The equations are solved along with appropriate boundary conditions, for a geometry based on the ultrasonic nebuliser, using the general purpose finite element software package Fastflo. The problem is axi-symmetric, with a curved (spherical) boundary representing the oscillating transducer.

For this example, we have taken $A=0.1 \mu \mathrm{m}$ and $\omega=1.2 \times 10^{7}$, which corresponds to a frequency of just under $2 \mathrm{MHz}$, typical of ultrasonic nebulisers. The corresponding wavelength of the ultrasound is $\lambda \approx 785 \mu \mathrm{m}$ and the length scale $L=125 \mu \mathrm{m}$. The geometry shown has an outer radius $60 L$, which means it has an outer diameter of $120 \mathrm{~L}=15 \mathrm{~mm}$. The actual nebuliser investigated during the MISG had a diameter of about $50 \mathrm{~mm}$, so the model geometry is much smaller. A finite element mesh comprising 11773 nodes and 5774 six-noded triangular elements with quadratic basis functions was used. A relatively fine mesh is required across the whole geometry, in order to accurately resolve the solutions, with several elements per wavelength necessary, as shown in Figure 1. In this case, there are around 10 nodes per wavelength. This is the reason that the overall scale of the geometry has been reduced from the true situation. It would be necessary to have a much larger mesh, and hence extremely long computation time to perform the calculations 


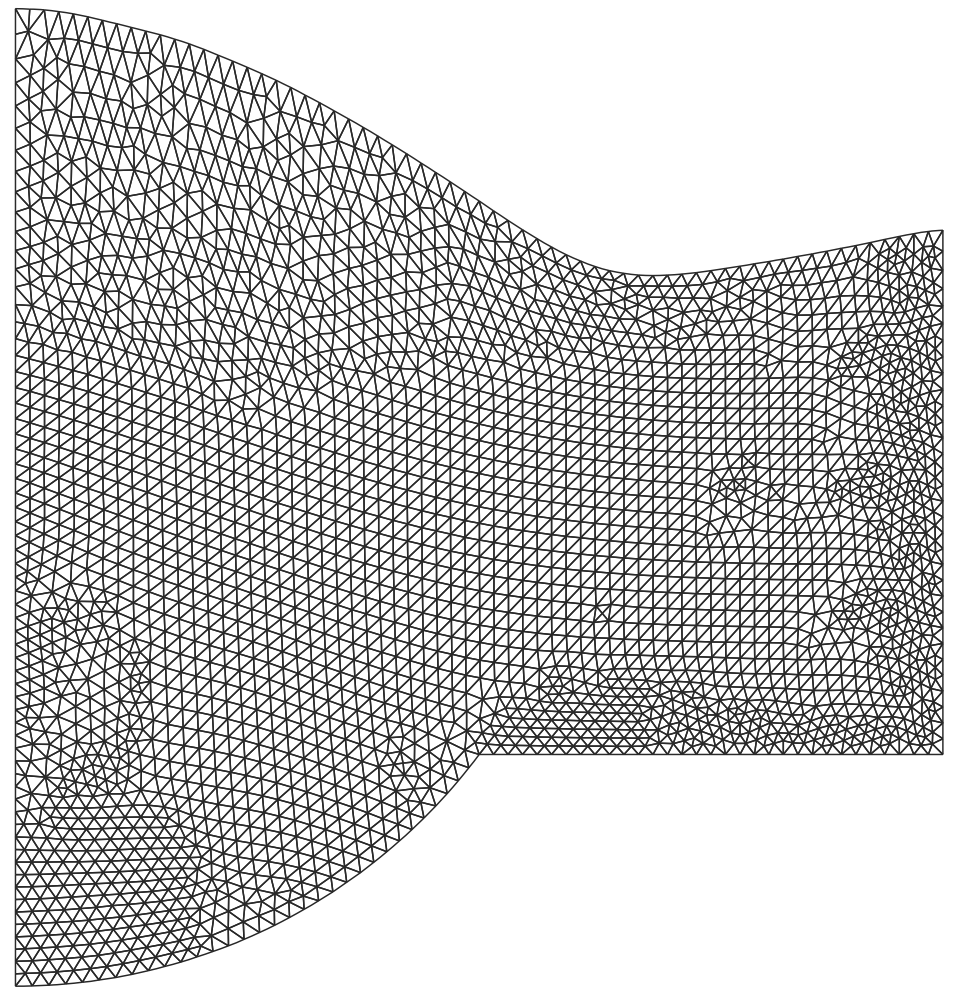

Figure 1: Finite element mesh. The problem is axisymmetric, so the left hand edge represents the centreline. 
for the full size geometry.

A pseudo time-stepping approach is used to adjust the free surface until a steady state is reached. At each time step, the linear equations for $\mathbf{u}_{s, c}$ are solved and then substituted into the flow equations (10-11) which are solved using an Augmented Lagrangian method. The mesh is moved along with the free surface, by solving a laplace equation for the vertical increment at any position, with zero movement on the bottom boundary, the free surface increment at the top boundary, and natural conditions on the side boundaries. This procedure ensures that the mesh does not deform too much, although there is clearly some stretching of elements near the free surface apparent in Figure 1, which shows the mesh at steady state.

In the results shown, $\mathrm{Re}_{c}=18.75$ and $\mathrm{Re}=0.015$ which are several orders of magnitude larger than the values arising from viscous dissipation, in order to account for the other dissipative effects mentioned previously.

Figure 2 shows contours of the calculated components of $\mathbf{u}_{s, c}$ when the solution scheme reaches a steady state. They show clearly the effects of the large dissipation, since very little of the acoustic signal remains near the free surface. Without the dissipative effect, the signal would reach the free surface and reflect back into the fluid, leading to a significantly different acoustic field. Figure 3(a) shows streamlines, calculated from the solution for $\mathbf{u}_{2}$ at steady state. They show a circulating flow, upwards in the centre, which is the cause of the bump on the free surface. This velocity profile only exists because of the dissipation effects. It is straightforward to show that, without the dissipation, the solution of the time independent equations would be $\mathbf{u}_{2}=0$. The circulating flow caused by the dissipation is an example of acoustic streaming. In this calculation, the focus is located below the mean free surface level. The steady pressure field $\phi_{2}+$ Fr $z$ is shown in Figure 3(b). This has been 


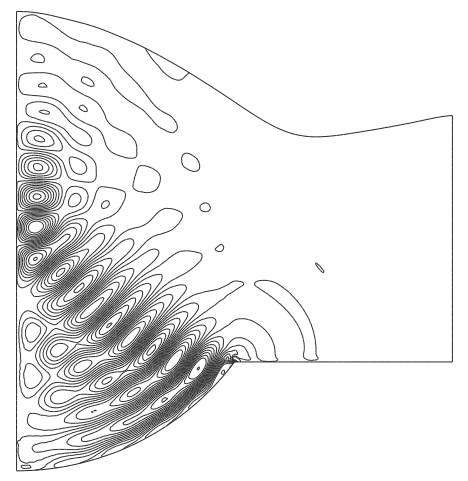

(a)

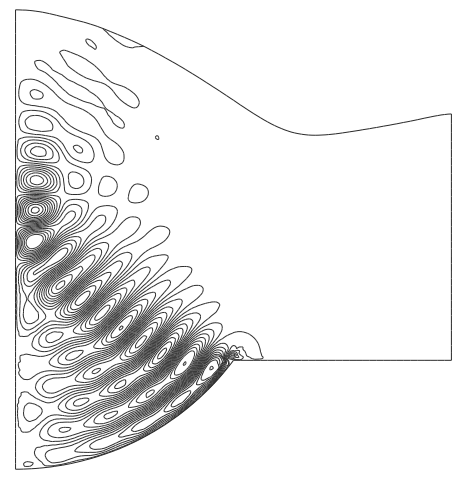

(c)



(b)

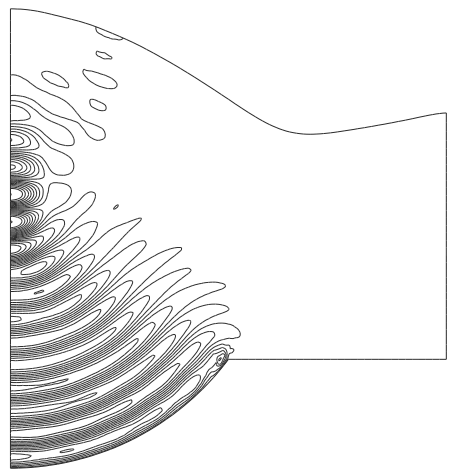

(d)

FiguRE 2: contours of (a) the radial component of $\mathbf{u}_{s}$ (b) the vertical component of $\mathbf{u}_{s}$, (c) the radial component of $\mathbf{u}_{c}$ and (d) the vertical component of $\mathbf{u}_{c}$. The curved part of the bottom represents the oscillating transducer. 


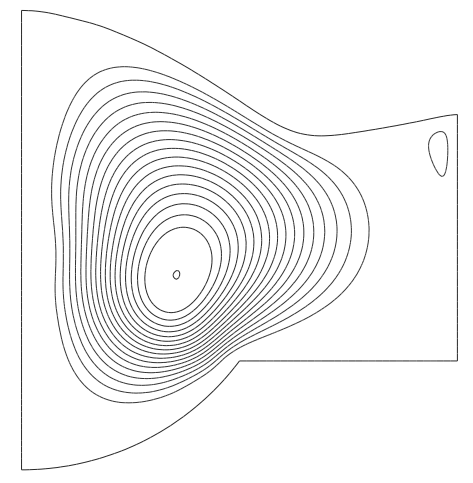

(a)

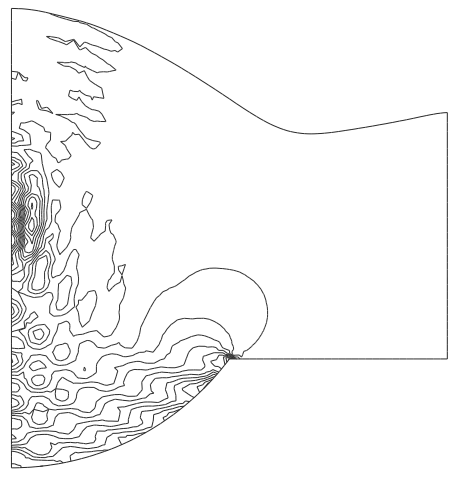

(b)

Figure 3: Contours of (a) a streamfunction derived from the solution for $\mathbf{u}_{2}$ and (b) pressure $\phi_{2}+\operatorname{Fr} z$.

corrected to remove the component of pressure contributed by the hydrostatic head, although it is convenient to solve the equations in the original form in order to correctly adjust the free surface. It is somewhat surprising that the pressure field exhibits such an oscillatory form, when the flow field is so smooth. However, consider the situation when there is no dissipation. In that case, $\phi_{2}$ would be a hydrostatic pressure that exactly balances the force terms on the right hand side of equation (11), which are oscillatory in nature. In the actual situation, with dissipation included, then $\phi_{2}$ is that hydrostatic pressure augmented by the flow effect.

Figure 4 shows curves of the steady free surface shapes calculated for different mean free surface levels. They show that the hump steepens as the level is lowered. If the level is lowered much more, a steady free surface shape was not able to be found. The procedure used to obtain the solution for each level was to begin with the 




FiguRE 4: Free surface shapes for two different depths of liquid in the ultrasonic nebuliser model. The dotted line has a mean depth 10 dimensionless units less than the solid line. For comparison, the curves have been plotted with the same mean value.

shape from the previous level, then lower the whole surface by a constant amount. Once the surface becomes too low, the centreline is "sucked" downwards which quickly leads to the blow-up of the solution procedure. It may be that this effect is a physical one, and that this type of behaviour is the cause of the splashing "fountain" effect observed in the real ultrasonic nebuliser.

Most of the cavitation will occur near the focus of the ultrasound. It is worth noting that the minima of $\phi_{2}$ are all located just off the centreline, whereas the maxima are located on the centre- 
line. This implies that the bulk of the cavitation bubbles would be formed away from the centreline. Cavitation bubbles that grow in this region will be advected by the flow, carrying them away from the centreline before they collapse. This effect, combined with buoyancy, should mean that the majority of bubbles reach the surface off the centreline. The current understanding of the mist formation process for ultrasonic nebulisers is that cavitation bubbles interact with the free surface to create liquid droplets [2]. A peculiar feature of the nebuliser considered at the MISG was that the mist formation occurred almost exclusively at a location off the centreline. The nature of the pressure field $\phi_{2}$ may help to explain this phenomenon. It would be an interesting, but challenging, task to develop a model of the growth, advection and collapse of cavitation bubbles in such a system.

There is the possibility to use the different parameters in the model to control the shape of the free surface. Increasing the transducer amplitude will increase the energy input to the system. Similarly, reducing the dissipation will lead to a less stable solution. Experience shows that travelling wave like solutions may be obtained if the dissipation is reduced. If it is further reduced, then it becomes impossible to obtain any steady solution. Of course, the wavelength of the ultrasound will also affect the behaviour.

\section{Conclusion}

We have shown that acoustic streaming, caused by the dissipative mechanisms occurring in an ultrasonic nebuliser, causes the formation of a bump on the free surface of a liquid, immediately above the focus of the ultrasound. Finally, advection of cavitation bubbles by the acoustic streaming flow provides an explanation for the location 
of the formation of the mist offset from the focal line. This work represents an initial attempt to understand the fluid dynamics of the ultrasonic nebuliser, and as such has a number of limitations. It would be desirable to carry out computations for a geometry with dimensions that are closer to the true geometry of an ultrasonic nebuliser. However, the calculations required to do this are large and beyond the scope of the current work, whose main aim is to demonstrate an effect, rather than closely simulate the real behaviour. In any case, the free surface shape in the real nebuliser geometry is violently unstable in the vicinity of the fountain formation. It would be difficult to properly represent such a situation. Finally, the approach used here in lumping all of the dissipative effects into an enhanced viscosity needs to be modified to more accurately represent the effect of cavitation, which is likely to be localised in the vicinity of the ultrasound focus. It would appear that a good model of the behaviour in an ultrasonic nebuliser remains a significant challenge.

\section{References}

[1] K. M. G. Taylor \& O. N. M. McCallion (1997) Ultrasonic nebulisers for pulmonary drug delivery. Int. J. Pharmaceutics, 153, 93-104. C465, C466

[2] G. Fulford \& G. Hocking (1998) Optimisation of an ultrasonic nebuliser. Proc 1998 Mathematics-in-Industry Study Group, Brisbane Australia, Feb 1998. C465, C476

[3] J. Lighthill (1978) Acoustic streaming, J. Sound Vib, 61(3), 391-418. C466 
[4] R. D. James \& J. W. Jacobs (1996) A round turbulent jet produced by an oscillating diaphragm. Phys. Fluids, 8(9), 2484-95. C466 